Received: 17 February 2017

Accepted: 18 May 2017

Published online: 07 July 2017

\section{On Local Unitary Equivalence of Two and Three-qubit States}

\author{
Bao-Zhi Sun ${ }^{1}$, Shao-Ming Fei ${ }^{2}$ \& Zhi-Xi Wang ${ }^{2}$
}

We study the local unitary equivalence for two and three-qubit mixed states by investigating the invariants under local unitary transformations. For two-qubit system, we prove that the determination of the local unitary equivalence of 2-qubits states only needs 14 or less invariants for arbitrary two-qubit states. Using the same method, we construct invariants for three-qubit mixed states. We prove that these invariants are sufficient to guarantee the LU equivalence of certain kind of three-qubit states. Also, we make a comparison with earlier works.

Nonlocality is one of the astonishing phenomena in quantum mechanics. It is not only important in philosophical considerations of the nature of quantum theory, but also the key ingredient in quantum computation and communications such as cryptography ${ }^{1}$. From the point of view of nonlocality, two states are completely equivalent if one can be transformed into the other by means of local unitary (LU) transformations. Many crucial properties such as the degree of entanglement ${ }^{2,3}$, maximal violations of Bell inequalities ${ }^{4-7}$ and the teleportation fidelity ${ }^{8,9}$ remain invariant under LU transformations. For this reason, it has been a key problem to determine whether or not two states are LU equivalent.

There have been a plenty of results on invariants under LU transformations ${ }^{10-25}$. However, one still does not have a complete set of such LU invariants which can operationally determine the LU equivalence of any two states both necessarily and sufficiently, except for 2-qubit states and some special 3-qubit states. For the 2-qubit state case, Makhlin presented a set of 18 polynomial LU invariants in ref. 10. In ref. 20 the authors constructed a set of very simple invariants which are less than the ones constructed in ref. 10. Nevertheless, the conclusions are valid only for special (generic) two-qubit states and an error occurred in the proof. In this paper, we corrected the error in ref. 20 by adding some missed invariants, and prove that the determination of the local unitary equivalence of 2-qubits states only needs 14 or less invariants for arbitrary two-qubit states. Moreover, we prove that the invariants in ref. 20 plus some invariants from triple scalar products of certain vectors are complete for a kind of 3-qubit states.

\section{Results}

A general 2-qubit state can be expressed as:

$$
\rho=\frac{1}{4}\left(I_{2} \otimes I_{2}+\sum_{i=1}^{3} T_{1}^{i} \sigma_{i} \otimes I_{2}+\sum_{j=1}^{3} T_{2}^{j} I_{2} \otimes \sigma_{j}+\sum_{i, j=1}^{3} T_{12}^{i j} \sigma_{i} \otimes \sigma_{j}\right),
$$

where $I$ is the $2 \times 2$ identity matrix, $\sigma_{i}, i=1,2,3$, are Pauli matrices and $T_{1}^{i}=\operatorname{tr}\left(\rho\left(\sigma_{i} \otimes I\right)\right)$ etc. Two two-qubit states $\rho$ and

$$
\hat{\rho}=\frac{1}{4}\left(I_{2} \otimes I_{2}+\sum_{i=1}^{3} \hat{T}_{1}^{i} \sigma_{i} \otimes I_{2}+\sum_{j=1}^{3} \hat{T}_{2}^{j} I_{2} \otimes \sigma_{j}+\sum_{i, j=1}^{3} \hat{T}_{12}^{i j} \sigma_{i} \otimes \sigma_{j}\right)
$$

are called LU equivalent if there are some $U_{i} \in U(2), i=1,2$, such that $\hat{\rho}=\left(U_{1} \otimes U_{2}\right) \rho\left(U_{1}^{\dagger} \otimes U_{2}^{\dagger}\right)$. By using the well-known double-covering map $S U(2) \rightarrow S O(3)$, one has that for all $U \in S U(2)$, there is a matrix $O=\left(o_{k l}\right) \in S O(3)$, such that $U \sigma_{k} U^{\dagger}=\sum_{l=1}^{3} o_{k l} \sigma_{l}$. Therefore, $\rho$ and $\hat{\rho}$ are LU equivalent if and only if there are some $O_{i} \in S O(3), i=1$, 2 , such that 


$$
\begin{aligned}
& \hat{T}_{1}=O_{1} T_{1}, \quad \hat{T}_{2}=O_{2} T_{2}, \\
& \hat{T}_{12}=O_{1} T_{12} O_{2}^{t} .
\end{aligned}
$$

One has two sets of vectors,

$$
\begin{aligned}
& S_{1}=\left\{T_{1}, T_{12} T_{2}, T_{12} T_{12}^{t} T_{1}, T_{12} T_{12}^{t} T_{12} T_{2}, \cdots\right\}, \\
& S_{2}=\left\{T_{2}, T_{12}^{t} T_{1}, T_{12}^{t} T_{12} T_{2}, T_{12}^{t} T_{12} T_{12}^{t} T_{1}, \cdots\right\} .
\end{aligned}
$$

For convenience, we denote $S_{1}=\left\{\mu_{i} \mid i=1,2, \cdots\right\}, S_{2}=\left\{\nu_{i} \mid i=1,2, \cdots\right\}$, i.e., $\mu_{1}=T_{1}, \mu_{2}=T_{12} T_{2}, \mu_{3}=T_{12} T_{12}^{t} T_{1}$ and so on. The vectors $\mu_{i}\left(v_{i}\right)$ are transformed into $O_{1} \mu_{i}\left(O_{2} v_{i}\right)$ under local unitary transformations. Otherwise, local unitary transformation can transform $\mu_{i} \times \mu_{j}$ to $O_{1}\left(\mu_{i} \times \mu_{j}\right)$ and $v_{i} \times v_{j}$ to $O_{2}\left(v_{i} \times v_{j}\right)$. Hence it is direct to verify that the inner products $\left\langle\mu_{i}, \mu_{j}\right\rangle,\left\langle v_{i}, v_{j}\right\rangle, i, j=1,2, \cdots$, and $\left(\mu_{i}, \mu_{j}, \mu_{k}\right) \equiv\left\langle\mu_{i}, \mu_{j} \times \mu_{k}\right\rangle,\left(\nu_{i}, \nu_{j}, \nu_{k}\right) \equiv$ $\left\langle\nu_{i}, \nu_{j} \times \nu_{k}\right\rangle, i, j, k=1,2, \cdots$, are invariants under local unitary transformations. Moreover, from the transformation $T_{12} \rightarrow O_{1} T_{12} O_{2}^{t}$, we have that $\operatorname{tr}\left(T_{12} T_{12}^{t}\right)^{\alpha}, \alpha=1,2, \cdots$, and det $T_{12}$ are also LU invariants.

For a set of 3-dimensional real vectors $S=\left\{\mu_{i} \mid i=1,2, \cdots\right\}$, we denote $\operatorname{dim}\langle S\rangle$ the dimension of the real linear space spanned by $\left\{\mu_{i}\right\}$, i.e., the number of linearly independent vectors of $\left\{\mu_{i}\right\}$. As the vectors in $S_{1}$ and $S_{2}$ are three-dimensional, there are at most 3 linearly independent vectors in each vector sets $S_{1}$ and $S_{2}$.

First note that, given two sets of 3-dimensional real vectors $S=\left\{\mu_{i} \mid i=1,2, \cdots\right\}$ and $\hat{S}=\left\{\hat{\mu}_{i} \mid i=1,2, \cdots\right\}$, if the inner products $\left\langle\mu_{i}, \mu_{j}\right\rangle=\left\langle\hat{\mu}_{i}, \hat{\mu}_{j}\right\rangle$, then the following conclusions are true: (i) $\operatorname{dim}\langle S\rangle=\operatorname{dim}\langle\hat{S}\rangle$; (ii) The corresponding subsets of $S$ and $\hat{S}$ have the same linear relations; (iii) There exist $O \in O(3)$ such that $\hat{\mu}_{i}=O \mu_{i}$. Furthermore, using $\left(\mu_{i}, \mu_{j}, \mu_{k}\right)=\left(\hat{\mu}_{i}, \hat{\mu}_{j}, \hat{\mu}_{k}\right)$, we can get that $O \in S O(3)$. If $\operatorname{dim}\langle S\rangle=3$, then $O$ is unique. For $\operatorname{dim}\langle S\rangle<3,\left(\mu_{i}, \mu_{j}, \mu_{k}\right)=\left(\hat{\mu}_{i}, \hat{\mu}_{j}, \hat{\mu}_{k}\right)=0$, and there is at least one $O \in S O(3)$ such that $\hat{\mu}_{i}=O \mu_{i}$.

Next we clarify the independent invariants in $S_{1}$ and $S_{2}$. From the definition of $\mu_{i}, v_{i}$, we have

$$
\begin{aligned}
& \left\langle\mu_{i}, \mu_{j}\right\rangle= \begin{cases}T_{1}^{t}\left(T_{12} T_{12}^{t}\right)^{a_{i j}} T_{1}, & \text { if } i, j \text { are odd } \\
T_{2}^{t}\left(T_{12}^{t} T_{12}\right)^{a_{i j}} T_{2}, & \text { if } i, j \text { are even } \\
T_{1}^{t}\left(T_{12} T_{12}^{t}\right)^{b_{i j}} T_{12} T_{2}, & \text { if } i+j \text { is odd }\end{cases} \\
& \left\langle\nu_{i}, \nu_{j}\right\rangle= \begin{cases}T_{2}^{t}\left(T_{12}^{t} T_{12}\right)^{a_{i j}} T_{2}, & \text { if } i, j \text { are odd } \\
T_{2}^{1}\left(T_{12} T_{12}^{t}\right)^{a_{i j}} T_{1}, & \text { if } i, j \text { are even } \\
T_{1}^{t}\left(T_{12} T_{12}^{t}\right)^{b_{i j}} T_{12} T_{2}, & \text { if } i+j \text { is odd }\end{cases}
\end{aligned}
$$

where $a_{i j}=(i+j-2) / 2, b_{i j}=(i+j-3) / 2$. From Hamilton-Cayley theorem, when $a_{i j}, b_{i j} \geq 3$, the invariants $\left\langle\mu_{i}, \mu_{j}\right\rangle$ and $\left\langle v_{i}, v_{j}\right\rangle$ can be linearly represented by $\left\langle\mu_{p}, \mu_{q}\right\rangle,\left\langle v_{p}, v_{q}\right\rangle, a_{p q}, b_{p q}<3$. Therefore there are only 9 linearly independent invariants: $\left\langle\mu_{i}, \mu_{j}\right\rangle,\left\langle v_{i}, v_{i}\right\rangle, i=1,2,3$, and $\left\langle\mu_{1}, \mu_{j}\right\rangle, j=2,4,6$. We denote them as $L=$ $L=\left\{\left\langle\mu_{i}, \mu_{i}\right\rangle,\left\langle\nu_{i}, \nu_{i}\right\rangle,\left\langle\mu_{1}, \mu_{j}\right\rangle \mid i=1,2,3, j=2,4,6\right\}$.

For 2-qubit states $\rho$ and $\hat{\rho}$, if $\operatorname{dim}\left\langle S_{1}\right\rangle=\operatorname{dim}\left\langle\hat{S}_{1}\right\rangle=3$, we need one more invariant $\left(\mu_{r_{0}}, \mu_{s_{0}}, \mu_{t_{0}}\right)$ to guarantee that there is an $O_{1} \in S O(3)$ such that $O_{1} \mu_{i}=\hat{\mu_{i}}$, for any $i$. Here $\mu_{r_{0}}, \mu_{s_{0}}$ and $\mu_{t_{0}}$ are arbitrary three linear independent vectors in $S_{1}$. If $\operatorname{dim}\left\langle S_{1}\right\rangle=\operatorname{dim}\left\langle\hat{S}_{1}\right\rangle<3$, then the invariants in $L$ are enough to guarantee the existence of $O_{1}$. Similar conclusions are true for $S_{2}$ and $\hat{S}_{2}$.

Let $\mu_{r_{0}}, \mu_{s_{0}}$ and $\mu_{t_{0}}\left(\nu_{r_{0}}, \nu_{s_{0}}\right.$ and $\left.\nu_{t_{0}}\right)$ denote arbitrary three linear independent vectors in $S_{1}\left(S_{2}\right)$ if $\operatorname{dim}\left\langle S_{1}\right\rangle=3$ $\left(\operatorname{dim}\left\langle S_{2}\right\rangle^{0}=3\right)$. For the case that at least one of $\operatorname{dim}\left\langle S_{1}\right\rangle$ and $\operatorname{dim}\left\langle S_{2}\right\rangle$ is 3, we have

Theorem 1 Two 2-qubit states are LU equivalent if and only if they have same values of the invariants in $L$, the invariant $\left(\mu_{r_{0}}, \mu_{s_{0}}, \mu_{t_{0}}\right)$ and/or $\left(\nu_{r_{0}}, \nu_{s_{0}}, \nu_{t_{0}}\right)$ if $\operatorname{dim}\left\langle S_{1}\right\rangle=3$ and/or $\operatorname{dim}\left\langle S_{2}\right\rangle=3$.

See Methods for the proof of Theorem 1 .

For the case both $\operatorname{dim}\left\langle S_{1}\right\rangle<3$ and $\operatorname{dim}\left\langle S_{2}\right\rangle<3$, we also have $O_{2} T_{12}^{t} \mu_{i}=\hat{T}_{12}^{t} O_{1} \mu_{i}$ for some $O_{i} \in S O(3)$. But this does not necessarily give rise to $\hat{T}_{12}=O_{1} T_{12} O_{2}^{t}$. In order to discuss these cases, we need the following result.

Lemma 1 For two-qubit states $\rho$ and $\hat{\rho}$, if $\operatorname{tr}\left(T_{12} T_{12}^{t}\right)^{\alpha}=\operatorname{tr}\left(\hat{T}_{12} \hat{T}_{12}^{t}\right)^{\alpha}, \alpha=1,2$ and $\operatorname{det} T_{12}=\operatorname{det} \hat{T}_{12}$. then $\hat{T}_{12}=$ $\mathrm{O}_{1} \mathrm{~T}_{12} \mathrm{O}_{2}^{t}$ for some $\mathrm{O}_{1}, \mathrm{O}_{2} \in \mathrm{SO}(3)$.

See Methods for the proof of Lemma 1.

For the completeness of the set of invariants, we also need an extra invariant $\mathbf{I}=\varepsilon_{i j k} \varepsilon_{l m n} T_{1}^{i} T_{2}^{l} T_{12}^{j m} T_{12}^{k n}$, here $\varepsilon_{i j k}$ and $\varepsilon_{l m n}$ are Levi-Cevita symbol. Now we discuss the case of $\operatorname{dim} S_{i}=\operatorname{dim} \hat{S}_{i}<3, i=1,2$.

Theorem 2 Two 2-qubit states with $\operatorname{dim} S_{i}=\operatorname{dim} \hat{S}_{i}<3, i=1,2$ are local unitary equivalent if and only if they have the same values of the invariants in $L$, and the invariants $\operatorname{tr}\left(T_{12} T_{12}^{t}\right)^{\alpha}, \alpha=1,2$, det $T_{12}$ and $I$.

See Methods for the proof of Theorem 2.

From Theorem 1 and 2 we see that for the case at least one of $\left\langle S_{i}\right\rangle$ has dimension three, we only need 11 or 10 invariants to determine the local unitary equivalence of two 2-qubit states: namely, 9 invariants from $L$, and $\left(\mu_{r_{0}}, \mu_{s_{0}}, \mu_{t_{0}}\right)$ and/or $\left(\nu_{r_{0}}, \nu_{s_{0}}, \nu_{t_{0}}\right)$. If both the dimensions of $\left\langle S_{1}\right\rangle$ and $\left\langle S_{2}\right\rangle$ are less than 3 , then $\left(\mu_{r_{0}}, \mu_{s_{0}}, \mu_{t_{0}}\right)=$ $\left(\nu_{r_{0}}, \nu_{s_{0}}, \nu_{t_{0}}\right)=0$. To determine the LU equivalence, we need invariants from $L, I, \operatorname{tr}\left(T_{12} T_{12}^{t}\right)^{\alpha}, \alpha=1,2$, and $\operatorname{det} T_{12}$. 
Hence we need at most 13 independent invariants. In ref. 20, the authors considered only the generic case of $\operatorname{dim}\left\langle S_{i}\right\rangle=3, i=1$ and 2 , in which the important invariants $\left(\mu_{r_{0}}, \mu_{s_{0}}, \mu_{t_{0}}\right)$ and $\left(\nu_{r_{0}}, \nu_{s_{0}}, \nu_{t_{0}}\right)$ are missed. By adding these missed invariants, we have remedied the error in ref. 20 and, moreover, generalized the method to the case of $\operatorname{dim}\left\langle S_{i}\right\rangle=3$ for $i=1$ or 2 .

As an example, let we consider the states $\rho$ and $\hat{\rho}$ with $T_{1}=(1,1,1)^{t}$ and $\hat{T}_{1}=(1,1,-1)^{t}$, respectively. $T_{2}=\hat{T}_{2}$ and $T_{12} T_{12}^{t}=\hat{T}_{12} \hat{T}_{12}^{t}$ are diagonal with different nonzero elements on diagonal line. Hence $\operatorname{dim}\left\langle S_{1}\right\rangle=\operatorname{dim}\left\langle\hat{S}_{1}\right\rangle=3$. In this case the invariants from ref. 20 have the same values for $\rho$ and $\hat{\rho}$. Nevertheless, taking $\mu_{r_{0}}=T_{1}, \mu_{s_{0}}=T_{1} T_{12} T_{12}^{t}$ and $\mu_{t_{0}}=T_{1}\left(T_{12} T_{12}^{t}\right)^{2}$, and correspondingly, $\hat{\mu}_{r_{0}}=\hat{T}_{1}, \hat{\mu}_{s_{0}}=\hat{T}_{1} \hat{T}_{12} \hat{T}_{12}^{t}$, and $\hat{\mu}_{t_{0}}=\hat{T}_{1}\left(\hat{T}_{12} \hat{T}_{12}^{t}\right)^{2}$, we find that the triple scalar invariant we added are different for $\rho$ and $\hat{\rho},\left(\mu_{r_{0}}, \mu_{s_{0}}, \mu_{t_{0}}\right)=-\left(\hat{\mu}_{r_{0}}, \hat{\mu}_{s_{0}}, \hat{\mu}_{t_{0}}\right) \neq 0$. Therefore, $\rho$ and $\hat{\rho}$ are not locally equivalent.

The expression of a complete set of LU invariants depends on the form of the invariants. Different constructions of LU invariants may give different numbers of the invariants in the complete set, and may have different advantages. Obviously the eigenvalues of a density matrix are LU invariants. Based on the eigenstate decompositions of density matrices, in ref. 12 complete set of LU invariants are presented for arbitrary dimensional bipartite states. Nevertheless, such kind of construction of invariants results in problems when the density matrices are degenerate, i.e. different eigenstates have the same eigenvalues. The $18 \mathrm{LU}$ invariants constructed in ref. 10 are based on the Bloch representations of 2-qubit states and has no such problem as in ref. 12. However, these 18 invariants are complete but more than necessary in the sense that the number of independent invariants can be reduced by suitable constructions of the invariants. The LU invariants constructed in ref. 20 are also in terms of Bloch representations. Such constructed invariants work for both non-degenerate and degenerate states. Nevertheless, the invariants: $\mathbf{I},\left(\mu_{r_{0}}, \mu_{s_{0}}, \mu_{t_{0}}\right),\left(\nu_{r_{0}}, \nu_{s_{0}}, \nu_{t_{0}}\right)$ and det $T_{12}=\operatorname{det} \hat{T}_{12}$ make the corresponding theorems incorrect even for generic cases studied in ref. 20. By adding these invariants, our set of invariants work for arbitrary 2-qubit states. In fact, a set of complete LU invariants characterizes completely the LU orbits in the quantum state space. Generally such orbits are not manifolds, but varieties. For example, the set of pure states is a symplectic variety ${ }^{26}$. For general mixed states, the situation is much more complicated ${ }^{27}$. Our results would highlight the analysis on the structures of LU orbits.

Now we come to discuss the case of three-qubit system. A three-qubit state $\rho$ can be written as:

$$
\begin{aligned}
\rho= & \frac{1}{8}\left(I_{2} \otimes I_{2} \otimes I_{2}+\sum_{i=1}^{3} T_{1}^{i} \sigma_{i} \otimes I_{2} \otimes I_{2}+\sum_{j=1}^{3} T_{2}^{j} I_{2} \otimes \sigma_{j} \otimes I_{2}+\sum_{k=1}^{3} T_{3}^{k} I_{2} \otimes I_{2} \otimes \sigma_{k}\right. \\
& +\sum_{i, j=1}^{3} T_{12}^{i j} \sigma_{i} \otimes \sigma_{j} \otimes I_{2}+\sum_{i, k=1}^{3} T_{13}^{i k} \sigma_{i} \otimes I_{2} \otimes \sigma_{k}+\sum_{j, k=1}^{3} T_{12}^{j k} I_{2} \otimes \sigma_{j} \otimes \sigma_{k} \\
& \left.+\sum_{i, j, k=1}^{3} T_{123}^{i j k} \sigma_{i} \otimes \sigma_{j} \otimes \sigma_{k}\right) .
\end{aligned}
$$

One has the coefficient vectors $T_{1}, T_{2}, T_{3}$, coefficient matrices $T_{12}, T_{23}, T_{13}$ and coefficient tensor $T_{123}$. Now, $\rho$ and $\hat{\rho}$ are LU equivalent if and only if there are $O_{i} \in S O(3), i=1,2,3$, such that $\hat{T}_{i}=O_{i} T_{i}, \hat{T}_{i j}=O_{i} \otimes$ $O_{j} T_{i j}, \hat{T}_{123}=O_{1} \otimes O_{2} \otimes O_{3} T_{123}$. For simplicity we denote $t_{i j k} \equiv T_{123}^{i j k}$ and

$$
\begin{aligned}
T_{1 \mid 23} & =\left(\begin{array}{lllllllll}
t_{111} & t_{112} & t_{113} & t_{121} & t_{122} & t_{123} & t_{131} & t_{132} & t_{133} \\
t_{211} & t_{212} & t_{213} & t_{221} & t_{222} & t_{223} & t_{231} & t_{232} & t_{233} \\
t_{311} & t_{312} & t_{313} & t_{321} & t_{322} & t_{323} & t_{331} & t_{332} & t_{333}
\end{array}\right), \\
T_{2 \mid 13} & =\left(\begin{array}{lllllllll}
t_{111} & t_{112} & t_{113} & t_{211} & t_{212} & t_{213} & t_{311} & t_{312} & t_{313} \\
t_{121} & t_{122} & t_{123} & t_{221} & t_{222} & t_{223} & t_{321} & t_{322} & t_{323} \\
t_{131} & t_{132} & t_{133} & t_{231} & t_{232} & t_{233} & t_{331} & t_{332} & t_{333}
\end{array}\right), \\
T_{3 \mid 12} & =\left(\begin{array}{lllllllll}
t_{111} & t_{121} & t_{131} & t_{211} & t_{221} & t_{231} & t_{311} & t_{321} & t_{331} \\
t_{112} & t_{122} & t_{132} & t_{212} & t_{222} & t_{232} & t_{312} & t_{322} & t_{332} \\
t_{113} & t_{123} & t_{133} & t_{213} & t_{223} & t_{233} & t_{313} & t_{323} & t_{333}
\end{array}\right) .
\end{aligned}
$$

Also, we write $\mathcal{T}_{1}=T_{1 \mid 23} T_{1 \mid 23}^{t}, \mathcal{T}_{2}=T_{2 \mid 13} T_{2 \mid 13}^{t}, \mathcal{T}_{3}=T_{3 \mid 12} T_{3 \mid 12}^{t}$ and $\mathcal{T}_{23}=T_{1 \mid 23}^{t} T_{1 \mid 23}, \mathcal{T}_{13}=T_{2 \mid 13}^{t} T_{2 \mid 13}, \mathcal{T}_{12}=$ $T_{3 \mid 12}^{t} T_{3 \mid 12}$. Similar to to the two-qubit case, one has three sets of vectors,

$$
\begin{aligned}
S_{1} & =\left\{\mathcal{T}_{1}^{r-1} T_{1}, \mathcal{T}_{1}^{r-1} T_{12} * *, \mathcal{T}_{1}^{r-1} T_{13} * *, \mathcal{T}_{1}^{r-1} T_{1 \mid 23} * *\right\}, \\
S_{2} & =\left\{\mathcal{T}_{2}^{r-1} T_{2}, \mathcal{T}_{2}^{r-1} T_{12}^{t} * *, \mathcal{T}_{2}^{r-1} T_{23} * *, \mathcal{T}_{2}^{r-1} T_{2 \mid 13} * *\right\}, \\
S_{3} & =\left\{\mathcal{T}_{3}^{r-1} T_{3}, \mathcal{T}_{3}^{r-1} T_{13}^{t} * *, \mathcal{T}_{3}^{r-1} T_{23}^{t} * *, \mathcal{T}_{3}^{r-1} T_{3 \mid 12} * *\right\},
\end{aligned}
$$

where $r=1,2,3$ and ** represents all the suitable vectors constructed from $T_{i j}, T_{i \mid j k}, \mathcal{T}_{i}$ and $T_{i}$ such that the vectors in $S_{i}$ are transformed into $O_{i} S_{i}$ under LU transformations. For instance, we have $T_{12}^{t} S_{1} \subset S_{2}$, $T_{13}^{t} S_{1} \subset S_{3}, T_{1 \mid 23} S_{2} \otimes S_{3} \subset S_{1}$ and so on, where for $S_{2}=\left\{\nu_{i} \mid i=1,2, \cdots\right\}$ and $S_{3}=\left\{\omega_{j} \mid j=1,2, \cdots\right\}$, we have denoted $S_{2} \otimes S_{3}=\left\{\nu_{i} \otimes \omega_{j} \mid i, j=1,2, \cdots\right\}$ etc. Because the vectors in $S_{i}$ are all 3 -dimensional, we have 
$\operatorname{dim}\left\langle S_{i}\right\rangle \leq 3$. The inner products $\left\langle\mu_{i}, \mu_{j}\right\rangle,\left\langle\nu_{i}, \nu_{j}\right\rangle$ and $\left\langle\omega_{i}, \omega_{j}\right\rangle, i, j=1,2, \ldots$, are all invariants under LU transformations. Using the method in ref. 20, we now prove that these invariants together with the additional ones in theorem 3 are sufficient to guarantee the LU equivalence of certain kind of three-qubit states with at least two of $\operatorname{dim}\left\langle S_{i}\right\rangle=3$ for $i=1,2,3$.

Theorem 3 Given two 3-qubit states $\rho$ and $\hat{\rho}$, if $\left\langle X_{i}, X_{j}\right\rangle=\left\langle\hat{X}_{i}, \hat{X}_{j}\right\rangle,\left(X_{i}, X_{j}, X_{k}\right)=\left(\hat{X}_{i}, \hat{X}_{j}, \hat{X}_{k}\right)$ for $X=\mu, \nu$, $\omega$ and $i, j, k=1,2, \cdots$, and $\operatorname{dim}\left\langle S_{i}\right\rangle=\operatorname{dim}\left\langle\hat{S}_{i}\right\rangle=3$ for at least two $i \in 1,2,3$, then $\rho$ and $\hat{\rho}$ are LU equivalent.

See Methods for the proof of Theorem 3.

If at most one of $\operatorname{dim}\left\langle S_{i}\right\rangle$ is 3 , things become more complicated. Now we give a comparison with the results in ref. 11. For 3-qubit states $\rho$ and $\hat{\rho}$, if

$$
\operatorname{tr}\left(\mathcal{T}_{i}^{r}\right)=\operatorname{tr}\left(\hat{\mathcal{T}}_{i}^{r}\right), \quad T_{i}^{t} \mathcal{T}_{i}^{r-1} T_{i}=\hat{T}_{i}^{t} \hat{\mathcal{T}}_{i}^{r-1} \hat{T}_{i}, \quad r, i=1,2,3,
$$

then there are $P_{i}, \hat{P}_{i} \in O(3)$ such that

$$
P_{i} \mathcal{T}_{i} P_{i}^{t}=\left(\begin{array}{ccc}
t_{i 1} & & \\
& t_{i 2} & \\
& & t_{i 3}
\end{array}\right)=\hat{P}_{i} \hat{\mathcal{T}}_{i} \hat{P}_{i}^{t}, \quad P_{i} T_{i}=\hat{P}_{i} \hat{T}_{i}=\left(\begin{array}{l}
a_{i 1} \\
a_{i 2} \\
a_{i 3}
\end{array}\right) .
$$

Denote

$$
Y_{i} \equiv\left(\begin{array}{ccc}
a_{i 1} & a_{i 2} & a_{i 3} \\
t_{i 1} a_{i 1} & t_{i 2} a_{i 2} & t_{i 3} a_{i 3} \\
t_{i 1}^{2} a_{i 1} & t_{i 2}^{2} a_{i 2} & t_{i 3}^{2} a_{i 3}
\end{array}\right)=\left(\begin{array}{ccc}
1 & 1 & 1 \\
t_{i 1} & t_{i 2} & t_{i 3} \\
t_{i 1}^{2} & t_{i 2}^{2} & t_{i 3}^{2}
\end{array}\right)\left(\begin{array}{lll}
a_{i 1} & & \\
& a_{i 2} & \\
& & a_{i 3}
\end{array}\right) \equiv \Lambda_{i} \Theta_{i} .
$$

The results in ref. 11 concluded that $\rho$ and $\hat{\rho}$ are local unitary equivalent if and only if the invariants in Theorem 3, together with the invariants $\operatorname{tr}\left(\mathcal{T}_{i}^{r}\right), r, i=1,2,3$ for the case of det $\Lambda_{i} \Theta_{i} \neq 0, i=1,2,3$. Obviously, if det $\Lambda_{i} \Theta_{i} \neq 0, P_{i} T_{i}, P_{i} \mathcal{T}_{i} T_{i}$ and $P_{i} \mathcal{T}_{i}^{2} T_{i}$ are linear independent, so all $\operatorname{dim}\left\langle S_{i}\right\rangle=3$. But $\operatorname{dim}\left\langle S_{i}\right\rangle=3$ does not necessarily imply det $\Lambda_{i} \Theta_{i} \neq 0$. Here we only need that two of the $\operatorname{dim}\left\langle S_{i}\right\rangle$ are 3 . So we give the sufficient conditions for local unitary equivalence of more states than the ones given in ref. 11.

\section{Conclusion}

We study the local unitary equivalence for two and three-qubit mixed states by investigating the invariants under local unitary transformations. We corrected the error in ref. 20 by adding some missed invariants, and prove that the determination of the local unitary equivalence of 2-qubits states only needs 14 or less invariants for arbitrary two-qubit states. Moreover, we prove that the invariants in ref. 20 plus some invariants from triple scalar products of certain vectors are complete for a kind of 3-qubit states. Comparing with the results in ref. 11, it has been shown that we judge the LU equivalence for a larger class of 3-qubit states.

\section{Methods}

Proof of Theorem 1 Suppose $\operatorname{dim}\left\langle S_{1}\right\rangle=\operatorname{dim}\left\langle\hat{S}_{1}\right\rangle=3$. From the construction of $S_{1}$ and $S_{2}$, we have that $\nu_{i+1}=T_{12}^{t} \mu_{i}, \hat{\nu}_{i+1}=\hat{T}_{12}^{t} \hat{\mu}_{i}, i=1,2, \cdots$. Then $O_{2} T_{12}^{t} \mu_{i}=O_{2} \nu_{i+1}=\hat{\nu}_{i+1}=\hat{T}_{12}^{t} \hat{\mu}_{i}=\hat{T}_{12}^{t} O_{1} \mu_{i}, i=1,2, \cdots$. Since $\mu_{r_{0}}, \mu_{s_{0}}$ and $\mu_{t_{0}}$ are linearly independent, $\operatorname{det}\left(\mu_{r_{0}} \mu_{s_{0}} \mu_{t_{0}}\right) \neq 0$, where $\left(\mu_{r_{0}} \mu_{s_{0}} \mu_{t_{0}}\right)$ denotes the $3 \times 3$ matrix given by the three column vectors $\mu_{r_{0}}, \mu_{s_{0}}$ and $\mu_{t_{0}}$. From $O_{2} T_{12}^{t}\left(\mu_{r_{0}} \mu_{s_{0}} \mu_{t_{0}}\right)=\hat{T}_{12}^{t} O_{1}\left(\mu_{r_{0}} \mu_{s_{0}} \mu_{t_{0}}\right)$, we get $O_{2} T_{12}^{t}=\hat{T}_{12}^{t} O_{1}$. Then $\hat{T}_{12}=O_{1} T_{12} O_{2}^{t}$. The same result can be obtained from $\operatorname{dim}\left\langle S_{2}\right\rangle=\operatorname{dim}\left\langle\hat{S}_{2}\right\rangle=3$.

Proof of Lemma 1 From $\operatorname{tr}\left(T_{12} T_{12}^{t}\right)^{\alpha}=\operatorname{tr}\left(\hat{T}_{12} \hat{T}_{12}^{t}\right)^{\alpha}, \alpha=1,2$ and det $T_{12}=\operatorname{det} \hat{T}_{12}$, one has that $T_{12}$ and $\hat{T}_{12}$ have the same singular values. According to the singular value decomposition, there are $P_{i}, \hat{P}_{i} \in O(3), i=1,2$, such that $P_{1} T_{12} P_{2}^{t}=\hat{P}_{1} \hat{T}_{12} \hat{P}_{2}^{t}=\operatorname{diag}\left(t_{1}, t_{2}, t_{3}\right)$, where $t_{1}, t_{2}$ and $t_{3}$ are the singular values. Set $O_{1}=\hat{P}_{1}^{t} P_{1}, O_{2}=\hat{P}_{2}^{t} P_{2} \in O(3)$, we have $T_{12}=O_{1} T_{12} O_{2}^{t}$. From det $T_{12}=\operatorname{det} \hat{T}_{12}$, we have that $\operatorname{det} O_{1}=\operatorname{det} O_{2}= \pm 1 . I f \operatorname{det} O_{1}=\operatorname{det} O_{2}=-1$, we may change $P_{i}$ to $-P_{i}$ to have $O_{i} \in S O(3)$.

Proof of Theorem 2 We only need to prove the "only if" part, i.e., to find $O_{1}, O_{2} \in S O(3)$ such that $\hat{T}_{12}=O_{1} T_{12} O_{2}^{t}, \hat{T}_{1}=O_{1} T_{1}$, and $\hat{T}_{2}=O_{2} T_{2}$ for two 2-qubit states $\rho$ and $\hat{\rho}$. From Lemma 1 , we have $P_{i}, \hat{P}_{i} \in O(3)$, such that $\hat{P}_{i}^{t} P_{i} \in S O(3)$ and

$$
P_{1} T_{12} P_{2}^{t}=\hat{P}_{1} \hat{T}_{12} \hat{P}_{2}^{t}=\operatorname{diag}\left(t_{1}, t_{2}, t_{3}\right)
$$

Hence

$$
P_{1} T_{12} T_{12}^{t} P_{1}^{t}=\hat{P}_{1} \hat{T}_{12} \hat{T}_{12}^{t} \hat{P}_{1}^{t}=P_{2} T_{12}^{t} T_{12} P_{2}^{t}=\hat{P}_{2} \hat{T}_{12}^{t} \hat{T}_{12} \hat{P}_{2}^{t}=\operatorname{diag}\left(t_{1}^{2}, t_{2}^{2}, t_{3}^{2}\right) .
$$

Let $D=\operatorname{diag}\left(t_{1}, t_{2}, t_{3}\right)$, then $P_{1} S_{1}=\left\{P_{1} T_{1}, D P_{2} T_{2}, D^{2} P_{1} T_{1}, D^{3} P_{2} T_{2}, D^{4} P_{1} T_{1}, \cdots\right\}, P_{2} S_{2}=\left\{P_{2} T_{2}, D P_{1} T_{1}, D^{2} P_{2} T_{2}\right.$, $\left.D^{3} P_{1} T_{1}, D^{4} P_{2} T_{2}, \cdots\right\}$, we have $\left\langle P_{1} \mu_{i}, P_{1} \mu_{j}\right\rangle=\left\langle\mu_{i}, \mu_{j}\right\rangle=\left\langle\hat{\mu}_{i}, \hat{\mu}_{j}\right\rangle=\left\langle\hat{P}_{1} \hat{\mu}_{i}, \hat{P}_{1} \hat{\mu}_{j}\right\rangle$, and $\left\langle P_{2} \nu_{i}, P_{2} \nu_{j}\right\rangle=\left\langle\hat{P}_{2} \hat{\nu}_{i}, \hat{P}_{2} \hat{\nu}_{j}\right\rangle$. Denote $P_{1} T_{1}=\left(\begin{array}{lll}a_{1} & b_{1} & c_{1}\end{array}\right)^{t}, P_{2} T_{2}=\left(\begin{array}{lll}a_{2} & b_{2} & c_{2}\end{array}\right)^{t}$. By using $\left\langle P_{1} \mu_{1}, P_{1} \mu_{j}\right\rangle=\left\langle\hat{P}_{1} \hat{\mu}_{1}, \hat{P}_{1} \hat{\mu}_{j}\right\rangle, j=1,3$, 5, i.e. $\left\langle P_{1} T_{1}, D^{r} P_{1} T_{1}\right\rangle=\left\langle\hat{P}_{1} \hat{T}_{1}\right.$, $\left.D^{r} \hat{P}_{1} \hat{T}_{1}\right\rangle, r=0,2$, 4, we get 


$$
t_{1}^{j} a_{1}^{2}+t_{2}^{j} b_{1}^{2}+t_{3}^{j} c_{1}^{2}=t_{1}^{j} \hat{a}_{1}^{2}+t_{2}^{j} \hat{b}_{1}^{2}+t_{3}^{j} \hat{c}_{1}^{2}, \quad j=0,2,4 .
$$

Similarly, using $\left\langle P_{2} \nu_{1}, P_{2} \nu_{j}\right\rangle=\left\langle\hat{P}_{2} \hat{\nu}_{1}, \hat{P}_{2} \hat{\nu}_{j}\right\rangle, j=1,3,5$, and $\left\langle P_{1} \mu_{1}, P_{1} \mu_{j}\right\rangle=\left\langle\hat{P}_{1} \hat{\mu}_{1}, \hat{P}_{1} \hat{\mu}_{j}\right\rangle, j=2,4,6$, we obtain

$$
\begin{gathered}
t_{1}^{j} a_{2}^{2}+t_{2}^{j} b_{2}^{2}+t_{3}^{j} c_{2}^{2}=t_{1}^{j} \hat{a}_{2}^{2}+t_{2}^{j} \hat{b}_{2}^{2}+t_{3}^{j} \hat{c}_{2}^{2}, \quad j=0,2,4 . \\
t_{1}^{j} a_{1} a_{2}+t_{2}^{j} b_{1} b_{2}+t_{3}^{j} c_{1} c_{2}=t_{1}^{j} \hat{a}_{1} \hat{a}_{2}+t_{2}^{j} \hat{b}_{1} \hat{b}_{2}+t_{3}^{j} \hat{c}_{1} \hat{c}_{2}, \quad j=1,3,5 .
\end{gathered}
$$

1. If $t_{1}, t_{2}, t_{3}$ are all not equal, from (6) and (7) we can conclude that $\alpha_{i}= \pm \hat{\alpha}_{i}$ for $\alpha=a, b, c$ and $i=1,2$.

(i) If $t_{i} \neq 0, i=1,2,3$, from (8) we get $\alpha_{1} \alpha_{2}=\hat{\alpha}_{1} \hat{\alpha}_{2}$ for $\alpha=a, b, c$. Now if $\alpha_{1} \alpha_{2} \neq 0$, then we have $\alpha_{1}=\hat{\alpha}_{1} \Leftrightarrow \alpha_{2}=\hat{\alpha}_{2}$. If $\alpha_{1} \alpha_{2}=0$, suppose $\alpha_{1}=0$, then we have $\hat{\alpha}_{1}=0$. If $\alpha_{2}=\hat{\alpha}_{2}$, we also can write $\alpha_{1}=\hat{\alpha}_{1}$. Let $R=\operatorname{diag}\left\{e_{1}, e_{2}, e_{3}\right\}$, where $e_{i}$ take values +1 or -1 , such that $R P_{1} T_{1}=\hat{P}_{1} \hat{T}_{1}$. Then one must have $R P_{2} T_{2}=\hat{P}_{2} \hat{T}_{2}$. Note that the equality (5) is also true if one replaces $P_{i}$ by $R P_{i}$. Let $O_{1}=\hat{P}_{1}^{t} R P_{1}, O_{2}=\hat{P}_{2}^{t} R P_{2}$. We have $\hat{T}_{i}=O_{i} T_{i}$ for $i=1,2$, and $\hat{T}_{12}=O_{1} T_{12} O_{2}^{t}$. To assure that $O_{i}$ be special, we have det $R=1$. Firstly, from $\operatorname{dim}\left\langle P_{i} S_{i}\right\rangle=\operatorname{dim}\left\langle S_{i}\right\rangle<3$, we have that $P_{i} T_{i}, D^{2} P_{i} T_{i}, D^{4} P_{i} T_{i}$ are linearly dependent. Then there is at least one $\alpha_{i}^{0} \in\left\{a_{i}, b_{i}, c_{i}\right\}$ that is zero. Hence if $P_{1} T_{1}$ and $D^{2} P_{1} T_{1}$ are linearly independent, we have that $\mathrm{D} P_{2} T_{2}$ can be linearly represented by $P_{1} T_{1}$ and $D^{2} P_{1} T_{2}$. Using $t_{1} t_{2} t_{3} \neq 0$ and supposing $a_{1}=0$, we get that $a_{2}$ is also zero. Now $e_{1}$ in $R$ can be chosen to be 1 or -1 freely. We can choose $e_{1}$ to assure that det $R=1$. Similarly, for the case that $P_{2} T_{2}$ and $\mathrm{DP}_{2} T_{2}$ are linear independent, we can also find $R$ which has determinate one. Lastly, if $P_{i} T_{i}$ and $D^{2} P_{i} T_{i}$ are linear dependent, then there are at least two members are zero in $\left\{a_{i}, b_{i}, c_{i}\right\}, i=1,2$. Therefore, there is an $\alpha \in\{a, b, c\}$ satisfying $\alpha_{1}=\alpha_{2}=0$, such that $\operatorname{det} R=1$.

(ii) If there exists a $t_{i}=0$, say, $t_{3}=0$, then we have $\alpha_{1} \alpha_{2}=\hat{\alpha}_{1} \hat{\alpha}_{2}$ for $\alpha=a, b$ from (8). And the invariant I can assure that $c_{1} c_{2}=\hat{c}_{1} \hat{c}_{2}$. From the discussion above, we have the conclusion.

2. If there are two different values of $t_{1}, t_{2}, t_{3}$, suppose $t_{1}=t_{2} \neq t_{3}$. Then from (6) and (7), we can get $a_{i}^{2}+b_{i}^{2}=\hat{a}_{i}^{2}+\hat{b}_{i}^{2}, c_{i}= \pm \hat{c}_{i}$ for $i=1,2$.

(i) If $t_{i} \neq 0, i=1,2,3$, from (8) we get $a_{1} a_{2}+b_{1} b_{2}=\hat{a}_{1} \hat{a}_{2}+\hat{b}_{1} \hat{b}_{2}, c_{1} c_{2}=\hat{c}_{1} \hat{c}_{2}$. Then there exists a matrix $M \in O(2)$ such that $M\left(\begin{array}{l}a_{i} \\ b_{i}\end{array}\right)=\left(\begin{array}{l}\hat{a}_{i} \\ \hat{b}_{i}\end{array}\right), i=1,2$. And there is an $e=1$ or -1 such that $e c_{i}=\hat{c}_{i}$ for $i=1,2$.

Therefore letting $R=\left(\begin{array}{ll}M & \\ & e\end{array}\right)$, one has $R P T_{1}=\hat{P} \hat{T}_{1}$ and $R Q T_{2}=\hat{Q} \hat{T}_{2}$ again. For the speciality of $R$, from the dimension of $\left\langle S_{i}\right\rangle$, we have $\operatorname{det}\left(\begin{array}{ll}a_{1} & a_{2} \\ b_{1} & b_{2}\end{array}\right)=0$ or $c_{1}=c_{2}=0$. Hence, we can choose suitable $M$ or $e$ to make sure that $R$ is special.

(ii) If $t_{1}=t_{2}=0$, we only have $c_{1} c_{2}=\hat{c}_{1} \hat{c}_{2}$. We can get $M_{i} \in O(2)$ such that $M_{i}\left(\begin{array}{l}a_{i} \\ b_{i}\end{array}\right)=\left(\begin{array}{l}\hat{a}_{i} \\ \hat{b}_{i}\end{array}\right), i=1,2$, and $R_{i}=\left(\begin{array}{ll}M_{i} & \\ & e\end{array}\right)$ to get the result similarly. We can choose suitable $M_{i}$ for the speciality of $R_{i}$.

(iii) If $t_{3}=0$, then one has $R_{1}, R_{2}$ with the same $M$ but different $e$ to prove the theorem. The speciality for $R_{i}$ is similar to the case of $t_{i} \neq 0$.

3. If $t_{1}=t_{2}=t_{3} \neq 0$, from (6), (7) and (8), we get $a_{i}^{2}+b_{i}^{2}+c_{i}^{2}=\hat{a}_{i}^{2}+\hat{b}_{i}^{2}+\hat{c}_{i}^{2}$ for $i=1,2$, and $a_{1} a_{2}+b_{1} b_{2}+c_{1} c_{2}=\hat{a}_{1} \hat{a}_{2}+\hat{b}_{1} \hat{b}_{2}+\hat{c}_{1} \hat{c}_{2}$. Then we have $R \in S O(3)$ such that $R P_{1} T_{1}=\hat{P}_{1} \hat{T}_{1}$ and $R P_{2} T_{2}=\hat{Q}_{2} \hat{T}_{2}$. Replacing $P_{i}$ by $R P_{i}$ in (5) we get the result.

4. If $t_{1}=t_{2}=t_{3}=0$, we have $a_{i}^{2}+b_{i}^{2}+c_{i}^{2}=\hat{a}_{i}^{2}+\hat{b}_{i}^{2}+\hat{c}_{i}^{2}$ for $i=1,2$. Therefore one has $R \in S O(3)$ such that $R P_{i} T_{i}=\hat{P}_{i} \hat{T}_{i}, i=1,2$. Replacing $P_{i}$ by $R P_{i}$ in (5) one gets the result.

Proof of Theorem 3 For 3-qubit states $\rho$ and $\hat{\rho}$, they are LU equivalent if and only if there are $O_{i} \in S O(3), i=1,2$, 3, such that $\hat{T}_{i}=O_{i} T_{i}, \hat{T}_{i j}=O_{i} T_{i j} O_{j}^{t}$ and $\hat{T}_{123}=O_{1} \otimes O_{2} \otimes O_{3} T_{123}$. Suppose $\operatorname{dim}\left\langle S_{i}\right\rangle=\operatorname{dim}\left\langle\hat{S}_{i}\right\rangle=3$, for $i=1,2$. By using the given invariants, we have $O_{i} \in S O(3)$ such that $\hat{\mu}_{i}=O_{1} \mu_{i}, \hat{\nu}_{i}=O_{2} \nu_{i}$ and $\hat{\omega}_{i}=O_{3} \omega_{i}$ for $i=1,2, \cdots$, as well as, $\hat{T}_{12}^{t} \hat{\mu}_{i}=O_{2} T_{12}^{t} \mu_{i}, \hat{T}_{13}^{t} \hat{\mu}_{i}=O_{3} T_{13}^{t} \mu_{i}, \hat{T}_{23}^{t} \hat{\nu}_{i}=O_{3} T_{23}^{t} \nu_{i}$ and $\hat{T}_{3 \mid 12} \hat{\mu}_{i} \otimes \hat{\nu}_{j}=O_{3} T_{3 \mid 12} \mu_{i} \otimes \nu_{j}$ for $i, j=1,2, \cdots$. Suppose $\mu_{i_{1}}, \mu_{i_{2}}$ and $\mu_{i_{3}}$ are linear independent. Then $O_{2} T_{12}^{t}\left(\mu_{i_{1}} \mu_{i_{2}} \mu_{i_{3}}\right)=\hat{T}_{12}^{t}\left(\hat{\mu}_{i_{1}} \hat{\mu}_{i_{2}} \hat{\mu}_{i_{3}}\right)=\hat{T}_{12}^{t} O_{1}\left(\mu_{i_{1}} \mu_{i_{2}} \mu_{i_{3}}\right)$. Hence we get $O_{2} T_{12}^{t}=\hat{T}_{12}^{t} O_{1}$, i.e. $\hat{T}_{12}=O_{1} T_{12} O_{2}^{t}$. Similarly, we have $\hat{T}_{13}=O_{1} T_{13} O_{2}^{t}, \hat{T}_{23}=O_{2} T_{23} O_{3}^{t}$. From $\hat{T}_{3 \mid 12} \hat{\mu}_{i} \otimes \hat{\nu}_{j}=O_{3} T_{3 \mid 12} \mu_{i} \otimes \nu_{j}, i, j=1,2, \cdots$, we have

$$
\hat{T}_{3 \mid 12} O_{1} \otimes O_{2}\left(\mu_{i_{1}} \mu_{i_{2}} \mu_{i_{3}}\right) \otimes\left(\nu_{j_{1}} \nu_{j_{2}} \nu_{j_{3}}\right)=O_{3} T_{3 \mid 12}\left(\mu_{i_{1}} \mu_{i_{2}} \mu_{i_{3}}\right) \otimes\left(\nu_{j_{1}} \nu_{j_{2}} \nu_{j_{3}}\right)
$$

where $\nu_{j_{1}}, \nu_{j_{2}}, \nu_{j_{3}}$ are linear independent vectors in $S_{2}$. Using the linear independence of $\mu_{i_{1}}, \mu_{i_{2}}, \mu_{i_{3}}$ and $\nu_{j_{1}}, \nu_{j_{2}}, \nu_{j_{3}}$, we get $\hat{T}_{3 \mid 12} O_{1} \otimes O_{2}=O_{3} T_{3 \mid 12}$ or $\hat{T}_{3 \mid 12}=O_{3} T_{3 \mid 12} O_{1}^{t} \otimes O_{2}^{t}$ which is equivalent to $T_{123}=$ $O_{1} \otimes O_{2} \otimes O_{3} T_{123}$ 


\section{References}

1. Nielsen, M. A. \& Chuang, I. L. Quantum Computation and Quantum Information. Cambridge University Press, Cambridge, England(2000).

2. Bennett, C. H., DiVincenzo, D. P., Smolin, J. A. \& Wootters, W. K. Mixed-state entanglement and quantum error correction. Phys. Rev. A 54, 3824 (1996); Bennett, C. H., Bernstein, H. J., Popescu, S. \& Schumacher, B. Concentrating Partial Entanglement by Local Operations. ibid. 53, 2046 (1996); Vedral, V., Plenio, M. B., Rippin, M. A. \& Knight, P. L. Quantifying Entanglement. Phys. Rev. Lett. 78, 2275 (1997).

3. Wootters, W. K. Entanglement of Formation of an Arbitrary State of Two Qubits. Phys. Rev. Lett. 80, 2245 (1998).

4. Horodecki, R., Horodecki, P. \& Horodecki, M. Violating Bell inequality by mixed spin-1/2 states: necessary and sufficient condition. Phys. Lett. A 200, 340 (1995).

5. Werner, R. F. \& Wolf, M. M. All-multipartite Bell-correlation inequalities for two dichotomic observables per site. Phys. Rev. A 64, $032112(2001)$.

6. Żukowski, M. \& Brukner, Č. Bells Theorem for General N-Qubit States. Phys. Rev. Lett. 88, 210401 (2002).

7. Li, M. \& Fei, S. M. Bell inequalities for multipartite qubit quantum systems and their maximal violation. Phys. Rev. A 86, 052119 (2012).

8. Horodecki, M., Horodecki, P. \& Horodecki, R. General teleportation channel, singlet fraction, and quasidistillation. Phys. Rev. A 60, 1888 (1999).

9. Albeverio, S., Fei, S. M. \& Yang, W. L. Optimal teleportation based on bell measurements. Phys. Rev. A 66, 012301 (2002).

10. Makhlin, Y. Nonlocal Properties of Two-Qubit Gates and Mixed States, and the Optimization of Quantum Computations. Quant. Info. Proc. 1, 243 (2002).

11. Linden, N., Popescu, S. \& Sudbery, A. Nonlocal Parameters for Multiparticle Density Matrices. Phys. Rev. Lett. 83, 243 (1999); Sun, B. Z., Fei, S. M., Li-Jost, X. Q. \& Wang, Z. X. A Note on Equivalence of Bipartite States under Local Unitary Transformations. J. Phys. A 39, L43-L47 (2006).

12. Zhou, C. Q., Zhang, T. G., Fei, S. M., Jing, N. H. \& Li-Jost, X. Q. Local unitary equivalence of arbitrary dimensional bipartite quantum states. Phys. Rev. A 86, 010303(R) (2012).

13. Zhang, T. G., Zhao, M. J., Li, M., Fei, S. M. \& Li-Jost, X. Q. Criterion of local unitary equivalence for multipartite states. Phys. Rev. A 88, 042304 (2013).

14. Liu, B., Li, J. L., Li, X. \& Qiao, C. F. Local Unitary Classification of Arbitrary Dimensional Multipartite Pure States. Phys. Rev. Lett. 108, 050501 (2012).

15. Li, M., Zhang, T. G., Fei, S. M., Li-Jost, X. Q. \& Jing, N. H. Local unitary equivalence of multiqubit mixed quantum states. Phys. Rev. A 89, 062325 (2014).

16. Martins, A. M. Necessary and sufficient conditions for local unitary equivalence of multiqubit states. Phys. Rev. A 91, 042308 (2015).

17. Li, J. L. \& Qiao, C. F. Classification of arbitrary multipartite entangled states under local unitary equivalence. J. Phys. A 46, 075301 (2013).

18. Jing, N. H., Li, M., Li-Jost, X. Q., Zhang, T. G. \& Fei, S. M. SLOCC invariants for multipartite mixed states. J. Phys. A 47, 215303 (2014).

19. Specht, W. Zur Theorie der Matrizen II, Jahresbericht Deutsch Math. Verein. Jber. Deutsch Math. Verein. 50, 19 (1940).

20. Jing, N. H., Fei, S. M., Li, M., Li-Jost, X. Q. \& Zhang, T. G. Local unitary invariants of generic multiqubit states. Phys. Rev. A 92, 022306 (2015).

21. Albeverio, S., Fei, S. M., Parashar, P. \& Yang, W. L. Nonlocal properties and local invariants for bipartite systems. Phys. Rev. A 68, $010303(2003)$.

22. Albeverio, S., Fei, S. M. \& Goswami, D. Local Invariants for a Class of Mixed States. Phys. Lett. A 340, 37 (2005).

23. Albeverio, S., Cattaneo, L., Fei, S. M. \& Wang, X. H. Equivalence of Tripartite Quantum States under Local Unitary Transformations. Int. J. Quant. Inform. 3, 603 (2005).

24. Kraus, B. Local Unitary Equivalence of Multipartite Pure States. Phys. Rev. Lett. 104, 020504 (2010).

25. Kraus, B. Local unitary equivalence and entanglement of multipartite pure states. Physical Review A 82(3) (2010).

26. Maciążek, T., Oszmaniec, M. \& Sawicki, A. How many invariant polynomials are needed to decide local unitary equivalence of qubit states? J. Math. Phys. 54, 092201 (2013); Sawicki, A. \& Kuś, M. Geometry of the local equivalence of states. J. Phys. A: Math. Theor. 44, 495301 (2011).

27. King, R. C., Welsh, T. A. \& Jarvis, P. D. The mixed two-qubit system and the structure of its ring of local invariants. J. Phys. A. 40, 10083 (2007)

\section{Acknowledgements}

We would like to thank the referee for pointing out a serious mistake in the initial draft of the paper. This work is supported by NSFC (Grant No. 11401339, 11675113), NSF of Shandong (No. ZR2014AQ027).

\section{Author Contributions}

B.-Z.S., S.-M.F. and Z.-X.W. wrote the main manuscript text. All of the authors reviewed the manuscript.

\section{Additional Information}

Competing Interests: The authors declare that they have no competing interests.

Publisher's note: Springer Nature remains neutral with regard to jurisdictional claims in published maps and institutional affiliations.

Open Access This article is licensed under a Creative Commons Attribution 4.0 International License, which permits use, sharing, adaptation, distribution and reproduction in any medium or format, as long as you give appropriate credit to the original author(s) and the source, provide a link to the Creative Commons license, and indicate if changes were made. The images or other third party material in this article are included in the article's Creative Commons license, unless indicated otherwise in a credit line to the material. If material is not included in the article's Creative Commons license and your intended use is not permitted by statutory regulation or exceeds the permitted use, you will need to obtain permission directly from the copyright holder. To view a copy of this license, visit http://creativecommons.org/licenses/by/4.0/.

(C) The Author(s) 2017 